\title{
EL SIGNO DE LA AORTA FLOTANTE
}

\author{
Drs. Pablo Cikutovic $M^{(1)}$, Cristián Varela $U^{(2)}$, María Carolina Carvajal $B^{(3)}$.
}

1. Residente de Radiología, Universidad Mayor, Departamento de Radiología, Clínica Dávila.

2. Radiólogo, Profesor Asistente, Facultad de Medicina, Universidad Mayor, Departamento de Radiología, Clínica Dávila.

3. Residente de Radiología, Universidad de los Andes, Departamento de Radiología, Clínica Dávila.

\section{THE FLOATING AORTA SIGN}

\begin{abstract}
The floating aorta sign is caused by an enlarged retroperitoneal lymph node mass which surrounds and displaces the abdominal aorta anteriorly, separating it from the lumbar spine without evidence of compression or aortic stenosis. Thus, the aorta seems to be floating inside a soft tissue mass. This radiologic sign is typically observed in lymphomas. The differential diagnosis includes testicular cancer metastases, primary retroperitoneal sarcomas, tuberculosis adenitis, and retroperitoneal fibrosis. Although this sign can be seen on ultrasound studies, it is best demonstrated and assessed through computed tomography and magnetic resonance imaging sequences.
\end{abstract}

Keywords: Lymph node mass, Lymphoma, Retroperitoneum.

Resumen: El signo de la aorta flotante es causado por voluminosos conglomerados ganglionares que rodean y desplazan hacia anterior a la aorta abdominal, separándola de la columna lumbar, sin evidencias de compresión o estenosis aórtica. De esta manera la aorta parece flotar en el espesor de una masa de partes blandas. Se observa característicamente en linfomas. El diagnóstico diferencial incluye metástasis de carcinoma testicular, sarcomas retroperitoneales primarios, adenitis tuberculosa y fibrosis retroperitoneal. Aunque este signo puede observarse en estudios ultrasonográficos, es mejor demostrado y evaluado con tomografía computada y resonancia magnética.

Palabras clave: Linfoma, Masa ganglionar, Retroperitoneo.

Cikutovic $\boldsymbol{P}$ y cols. El signo de la aorta flotante. Rev Chil Radiol 2010; 16(3): 154-158.

Correspondencia: Dr. Pablo A. Cikutovic M.

pablocikutovicm@gmail.com

Trabajo recibido el 22 de junio de 2010, aceptado para publicación el 16 de agosto de 2010.

\section{Aspecto imaginológico}

El signo de la aorta flotante ("Floating Aorta Sign")(1) representa el patrón de compromiso ganglionar retroperitoneal de algunos tumores, en particular del linfoma. Se caracteriza por crecimiento y formación de conglomerados ganglionares alrededor de la aorta abdominal, la que se desplaza hacia anterior y queda separada de la columna lumbar, sin identificar compresión o estenosis luminal ${ }^{(1,2)}$ (Figura 1). De esta manera, en tomografía computada (TC) y también en resonancia magnética (RM), se genera la impresión de que la aorta abdominal se encuentra inmersa o "flotando" dentro de una masa retroperitoneal de partes blandas (2) (Figura 2). Este aspecto rememora el "signo angiográfico", el que consiste en una estructura vascular pulmonar surcando una condensación. Fue inicialmente descrito como específico del carcinoma bronquioloalveolar, que se manifestaba como una consolidación en TC de tórax con medio de contraste intravenoso ${ }^{(3)}$. Dada la similitud morfológica, este signo ha sido utilizado por algunos autores como sinónimo del "signo de la aorta flotante"(1).

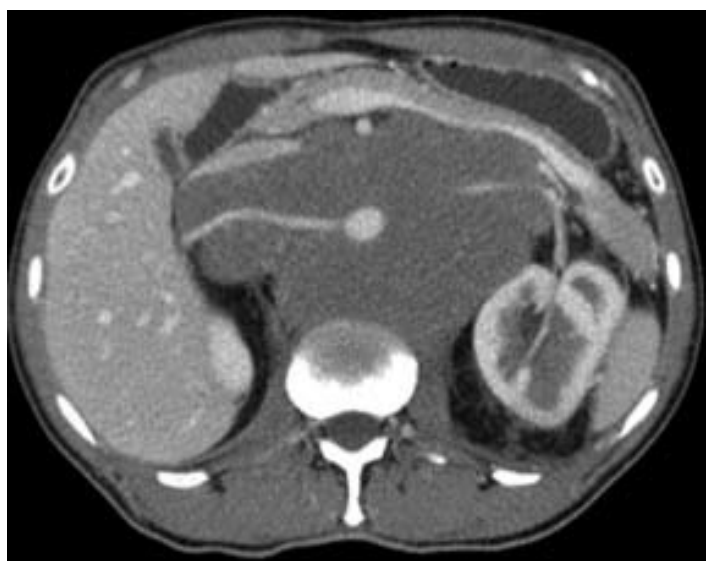

Figura 1. TC de abdomen con contraste intravenoso: corte axial a nivel de los hilios renales que demuestra signo de la "aorta flotante": aorta abdominal separada de la columna vertebral, "surcando" una gran masa ganglionar retroperitoneal homogénea que presenta discreto realce. 

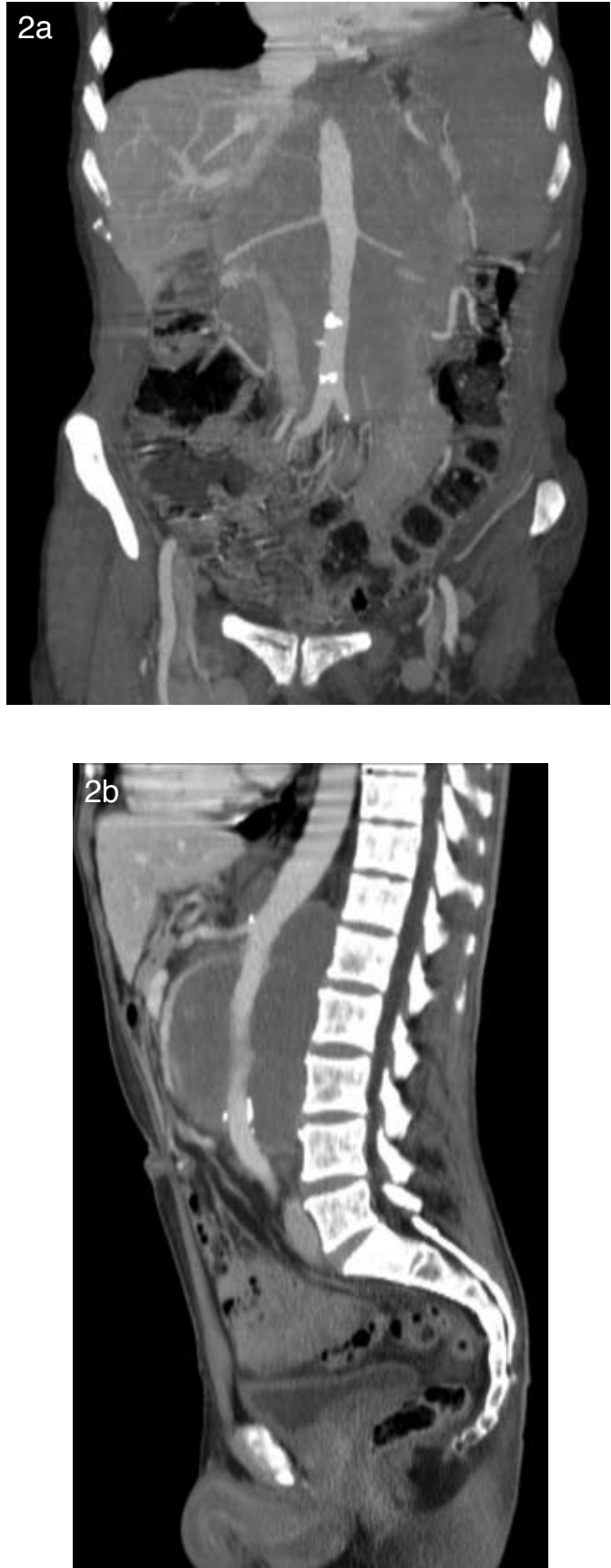

Figura 2. Signo de la "aorta flotante". (a) Reconstrucción coronal. (b) Reconstrucción sagital. La aorta abdominal infrarrenal impresiona atravesar la masa ganglionar.

\section{Significado}

Los linfonodos retroperitoneales presentan distribución perivascular, agrupándose en las cadenas periaórticas, pericavas e intercavoaorticas. Cuando estas cadenas son infiltradas por neoplasias, las adenopatías varían desde un leve aumento de tamaño hasta grandes masas confluentes; son estas últimas las que dan lugar al aspecto morfológico de la "aorta flotante". Es muy poco probable que un proceso benigno determine masas ganglionares tan voluminosas o confluentes como para generar el aspecto descrito.

\section{Diagnóstico diferencial}

Múltiples patologías pueden producir masas ganglionares retroperitoneales (Tabla I). El linfoma es la neoplasia maligna que con mayor frecuencia se presenta como una masa retroperitoneal(2), que está formada por múltiples y grandes adenopatías coalescentes, que característicamente forman una gran masa homogénea, con densidad de partes blandas y que se realza en forma discreta con el medio de contraste. A mayor tamaño, esta masa puede extenderse desde el nivel prevertebral hacia anterior y lateral abarcando las regiones paravertebrales $^{(4)}$, rodeando y desplazando la aorta abdominal hacia anterior.

Tabla I. Causas de masa ganglionar retroperitoneal.

- Linfoma

- Metástasis retroperitoneal

- Cáncer testicular

- Cáncer cervical

- Cáncer de mama

Diagnóstico diferencial

- Adenopatías infecciosas

- Fibrosis retroperitoneal

- Enfermedad de Castlemann

- Neoplasia maligna primaria

- Neoplasia benigna

- Tumor neuroendocrino

El signo de la "aorta flotante" es característico de los linfomas, por lo que su presencia sugiere el diagnóstico ${ }^{(2,5)}$. No es frecuente observar este aspecto en otras neoplasias metastásicas, así como tampoco en sarcomas mesenquimáticos o tumores neurogénicos ${ }^{(2)}$.

Una masa ganglionar puede reflejar la extensión linfática de un tumor desde un órgano de la región, como el carcinoma de células renales, o bien remoto, como una neoplasia testicular, cérvicouterina o prostática. De éstas, las neoplasias testiculares originan grandes masas ganglionares retroperitoneales que pueden desplazar la aorta hacia anterior, dando un aspecto similar al linfoma. Esta masa retroperitoneal puede presentar signos de necrosis o calcificaciones en su espesor ${ }^{(6)}$ (Figura 3). En ocasiones, las masas ganglionares metastásicas no desplazan las estructuras vasculares a pesar de alcanzar gran tamaño (Figura 4). 

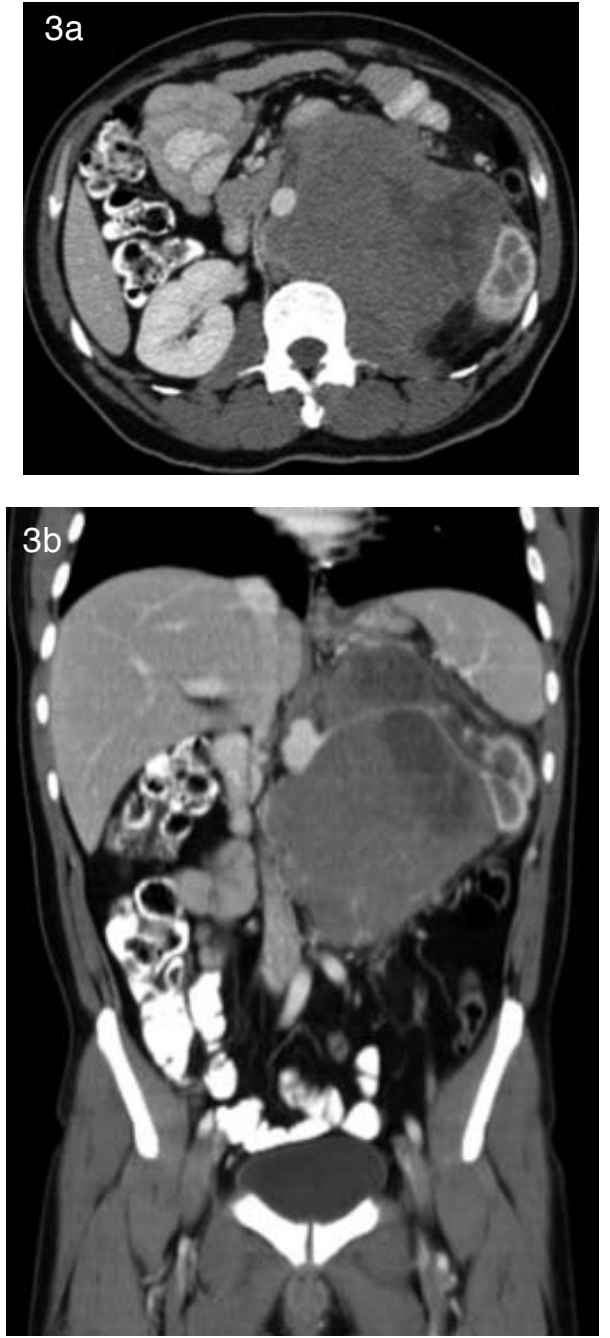

Figura 3. Metástasis ganglionares de cáncer testicular. TC de abdomen con contraste intravenoso que evidencia una masa retroperitoneal izquierda que se extiende hacia la línea media, desplazando la aorta abdominal hacia anterior, además elonga los vasos renales y comprime el riñón izquierdo. La masa posee distintas densidades con áreas que sugieren necrosis tumoral. (a) Corte axial (a). (b) Reconstrucción coronal.

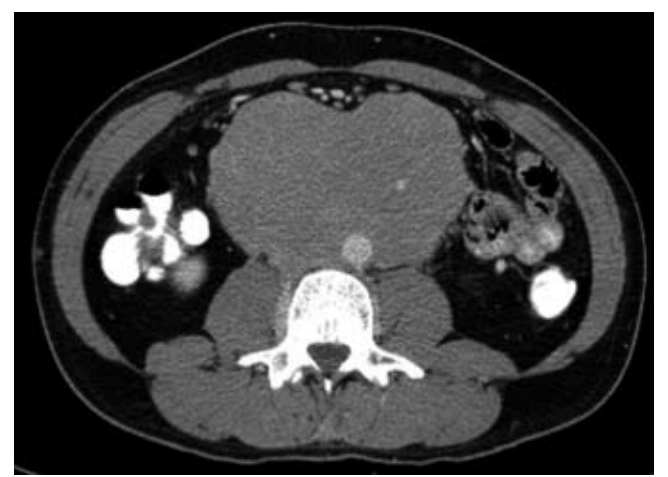

Figura 4. Metástasis ganglionares de cáncer testicular. TC de abdomen con contraste intravenoso: corte axial que evidencia una voluminosa masa retroperitoneal, que se dispone anterior a la aorta abdominal, sin separarla de la columna. Sí desplaza hacia anterior la arteria mesentérica inferior.
Con mucho menor frecuencia, una masa retroperitoneal puede corresponder a una neoplasia primaria retroperitoneal de estirpe mesenquimática(1) y en ese caso, el desplazamiento de los órganos retroperitoneales (marcado y en distintas direcciones) $)^{(7)}$ junto con ausencia de signos que indiquen un posible órgano de origen, sugieren un tumor retroperitoneal primario. También puede sospecharse un tumor primario de este compartimento cuando la lesión posee áreas de densidad grasa (como en el caso del liposarcoma)(8), compromete o emerge de la vena cava inferior y presenta focos de necrosis, como en el leiomiosarcoma ${ }^{(9)}$.

Dentro de las causas benignas, las de etiología infecciosa, como tuberculosis (TBC) o micobacterios atípicos, suelen presentar un compromiso más discreto de los linfonodos retroperitoneales, con adenopatías más pequeñas que no tienden a confluir ni a constituir grandes masas (Figura 5), a veces con un centro hipodenso.

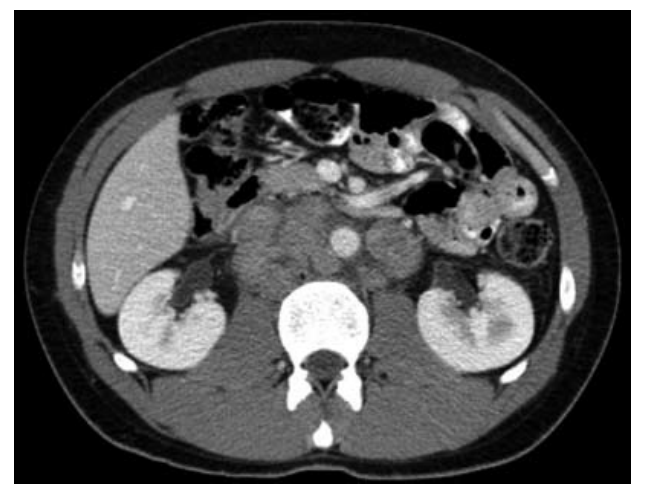

Figura 5. Infección por mycobacteria atípica. TC de abdomen con contraste intravenoso: corte axial a nivel de los hilios renales, que evidencia múltiples adenopatías retroperitoneales, algunas con centro hipodenso, que no tienden a confluir ni formar conglomerados. Además existe aumento de la densidad de la grasa periganglionar. La aorta abdominal presenta un discreto desplazamiento anterior.

La enfermedad de Castleman (hiperplasia angiofolicular) es una entidad poco común, que se manifiesta como conglomerados de adenopatías cervicales, mediastínicas o retroperitoneales, que característicamente captan ávidamente el contraste intravenoso ${ }^{(9)}$, a diferencia del linfoma, que es hipovascular.

Entre las enfermedades inflamatorias destaca la fibrosis retroperitoneal, que se presenta como una masa retroperitoneal de márgenes irregulares pero definidos, isodensa al músculo y que se dispone rodeando el aspecto anterior y lateral de la aorta infrarenal (Figura 6). Como regla, estas placas fibrótico-inflamatorias no desplazan hacia anterior la aorta abdominal ni la vena cava inferior ${ }^{(10)}$ y característicamente obstruyen los uréteres, observándose hidroureteronefrosis en TC (Figura 7), hallazgo infrecuente en el contexto de un linfoma. 

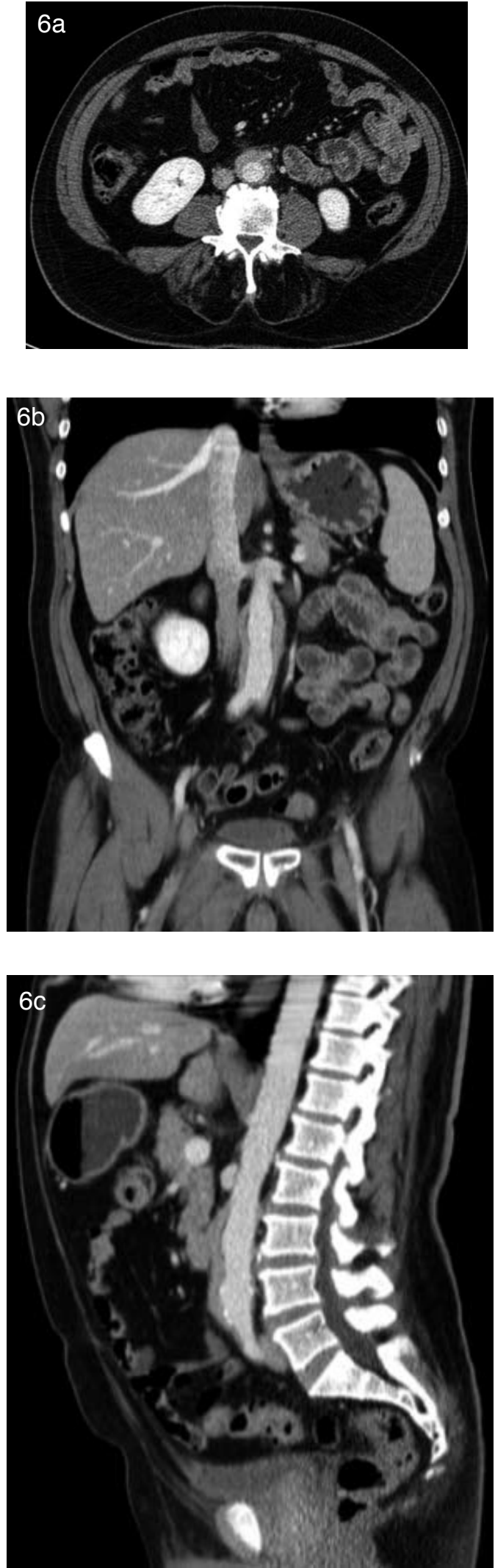

Figura 6. Fibrosis retroperitoneal. TC de abdomen con contraste intravenoso, fase portal, que evidencia una masa con densidad de partes blandas periaórtica, dispuesta anterior y lateral, sin comprometer la región retroaórtica ni producir desplazamiento de esta. (a) Corte axial. (b) Reconstrucción coronal. (c) Reconstrucción sagital.
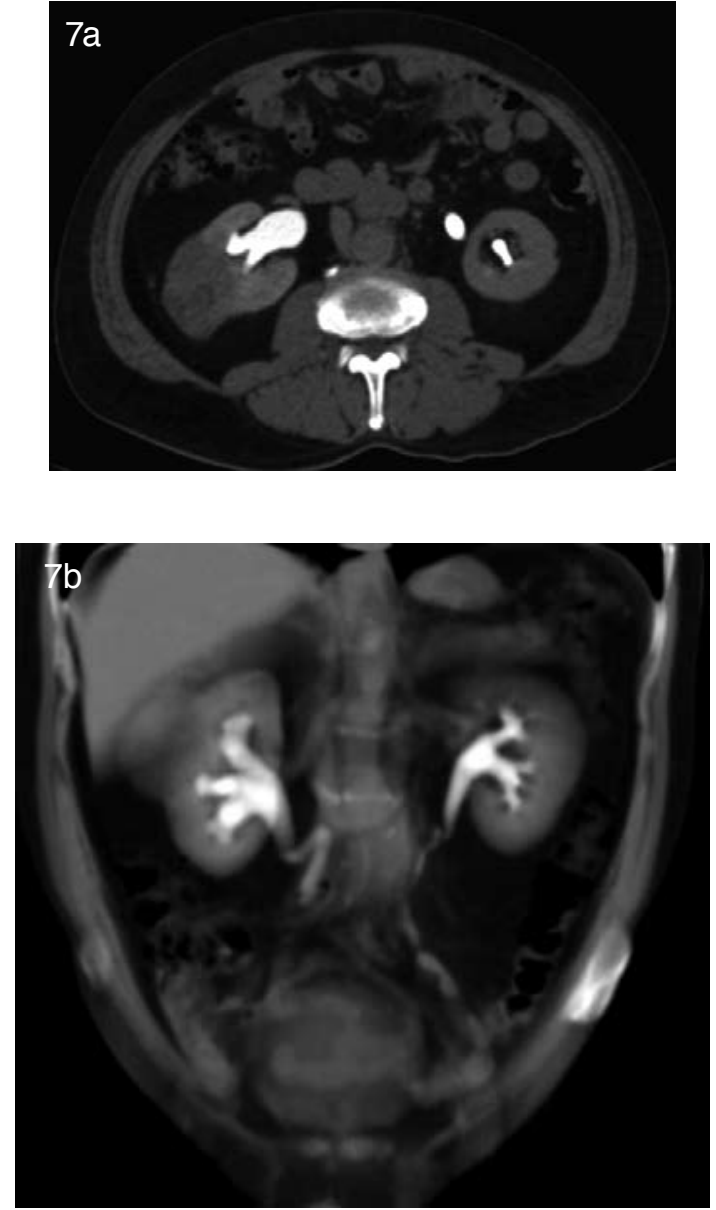

Figura 7. Fibrosis retroperitoneal. TC abdominal contrastado en fase de eliminación que evidencia fibrosis retroperitoneal que compromete ambos uréteres, más acentuado a derecha, determinando una hidronefrosis bilateral; característicamente los uréteres presentan estenosis extrínseca y desplazamiento medial. (a) Corte axial a nivel infrarrenal. (b) Reconstrucción coronal MIP.

\section{Discusión}

Los grandes avances en el desarrollo imaginológico actual han permitido que la TC y RM se constituyan en herramientas fundamentales para el estudio de las masas retroperitoneales. En el caso del linfoma, a diferencia de los tumores retroperitoneales primarios, resulta fundamental que el radiólogo sugiera el diagnóstico ya que una biopsia percutánea guiada por imágenes es lo más adecuado para estos pacientes y la cirugía no juega un rol importante en su manejo(2).

Es muy importante conocer el patrón de crecimiento de las masas retroperitoneales y los signos imaginológicos sugerentes de las posibles etiologías, con tal de constituir una guía en el diagnóstico diferencial y permitir un manejo clínico adecuado. 


\section{Bibliografía}

1. Nishino M, Hayakawa K, Minami M, Yamamoto A, Ueda H, Takasu K. Primary Retroperitoneal Neoplasms: CT and MR Imaging Findings with Anatomic and Pathologic Diagnostic Clues. RadioGraphics 2003; 23: 45-57.

2. Sanyal R, Remer E. Radiology of the Retroperitoneum: Self-Assessment Module. AJR 2009; 192: 118-121.

3. Maldonado R. The CT Angiogram Sign. Radiology 1999; 210: 323-324.

4. Toma P, Granata C, Rossi A, Garaventa A. Multimodality Imaging of Hodgkin Disease and Non-Hodgkin Lymphomas in Children. RadioGraphics 2007; 27: 1335-1354.

5. Vincent JM, Ng YY, Norton AJ, Armstrong P. CT "angiogram sign" in primary pulmonary lymphoma. J Comput Assist Tomogr 1992; 16: 829-831.

6. Sohaib S, Koh D, Husband J. The Role of Imaging in the Diagnosis, Staging, and Management of Testicular Cancer. AJR 2008; 191:387-395.

7. Lee JKT, Hiken JN, Semelka RC. Retroperitoneum. In: Lee JKT, Sagel SS, Stanley RJ, Heiken JP, eds. Computed body tomography with MRI correlation. 3rd ed. Philadelphia, Pa: Lippincott- Raven 1996; 1023-1086.

8. Kim EY, Kim SJ, Choi D, Lee SJ, Kim SH, Lim HK, Song SY. Recurrence of Retroperitoneal Liposarcoma: Imaging Findings and Growth Rates at Follow-Up CT. AJR 2008; 191: 1841-1846.

9. Pickhardt P. Peritoneum and Retroperitoneum. Slone R, Fisher A, Pickhardt P, Gutierrez F, Balfe D. Body CT, a practical aproach. 1rst ed, Mc Graw- Hill, 1999; 170-174.

10. Cronin C, Lohan D, Blake M, Roche C, McCarthy P, Murphy J. Retroperitoneal Fibrosis: A Review of Clinical Features and Imaging Findings. AJR 2008; 191: 423-431. 